\title{
An Approximate Solution of Fractional Kolmogorov-Petrovskii-Piskunov Equations
}

\author{
${ }^{1}$ Sumaira Yousuf Khan* and ${ }^{2}$ Sahar Altaf \\ ${ }^{1}$ College of Computer Science and Information Systems, Institute of Business Management \\ Korangi Creek, Karachi, Pakistan \\ ${ }^{2}$ College of Humanities and Sciences, PAF-KIET Karachi Institute of Economics and Technology, \\ Korangi Creek, Karachi, Pakistan \\ *Corresponding author: sumaira.khan@iobm.edu.pk
}

Article history

Received: 17 April 2019

Received in revised form: 26 October 2019

Accepted: 31 October 2019

Published online: 1 December 2019

\begin{abstract}
An approximate-analytical method known as Reduced Differential Transform Method (RDTM) is proposed to approximate the Fractional Kolmogorov-PetrovskiiPiskunov Equations. It is a powerful and convenient approximation analytical tool used for linear and nonlinear equations related to various science, engineering and industrial applications. Some illustrative examples are given to exemplify the competence of the proposed scheme and provide precise solutions for nonlinear problems.In addition, a comparison with the classical equation and Homotopy perturbation method illustrates the competency of the technique. The results show that the proposed technique requires minimum computational cost at rapid convergence. This method can be applied to other partial differential equations of fractional order for the analysis of their solutions.
\end{abstract}

Keywords Reduced differential transform method (RDTM); partial differential equations; space-time fractional Kolmogorov-Petrovskii-Piskunov (KPP) equations; Caputo derivative

Mathematics Subject Classification 33F05, 65D20, 35R11

\section{Introduction}

Partial differential equations have been considered an interesting topic among the researchers in the analysis of nonlinear phenomena related to the study of fluid mechanics, thermodynamics, heat transfer, Nano-hydro kinematics and others. In the past three decades, fractional calculus has gained much attention in several fields of science and engineering. The fractional order differential equations, specifically have a much broader spectrum of applications in complex models involving visco elasticity, liquid flow, and biological sciences, damping laws, diffusion processes and physical sciences [1-4]. However, an exact analytical solution to some fractional partial differential equations can be impractical. Thus, finding its numerical approach is productive. This issue has led to the establishments of several analytical approximate 
techniques in the analysis of fractional partial differential equations such as q-Homotopy Analysis technique [5], Homotopy Perturbation Method [6], Adomian Decomposition Technique [7], Variational Iteration Technique [8] and Reduced Differential Transform Method (RDTM). RDTM was first suggested by Keskin in his thesis [9] and applied in the approximation analysis of fractional partial differential equations [10] and [11]. Using this idea, numerous articles have been written in order to explore and handle the physical models that emerge in science and engineering [12-19].

In this study, we investigate one of the important types of reaction- diffusion equations namely, Kolmogrov-Petrovskii-Piskunov (KPP) equations of fractional order. The linear and nonlinear reaction-diffusion equations play active role in various models of reaction-diffusion, mathematical biology, chemistry, and genetics. Initially, KPP equations is used in the genetics model for the spread of an advantageous gene through a population. Later on, it has been applied to a number of physical, biological and chemical models which contain some well-known nonlinear equations in mathematical physics like the Newell-Whitehead equation, FitzHughNagumo equation, Huxley equation, Burgers- Huxley equation, the Fisher equation and ChaffeeInfant equation as special cases.

This paper is organized as follows. In section 2, we outline basic definitions and preliminaries of fractional calculus. In section 3, the key features of Fractional Reduced Differential Transform Method (FRDTM) are explained. Sections 4 and 5 are devoted to the methodology and implementation of the method on KPP equations respectively. Finally, section 6 discusses the conclusions of this study.

\section{Basic Theory of Fractional Calculus}

There are several definitions related to fractional order $\alpha>0$ in the literature, such as RiemannLiouville, Jumarie, Caputo and Riesz, Grunwald- Letnikov, Weyl, Hadamard and ErdelyiKober fractional derivatives [1-4]. Here, we revisit some fundamental definitions of the fractional calculus, which we utilize in this study.

Definition 1 Assume any function $\rho(x)$ in space $\mathrm{C}_{\mu}$ where $\mu \in \mathrm{R}$ and $x>0$. Also if a number $q$ exists where $q \in \mathbb{R}, q(>\mu)$ such that $\rho(x)=x^{q} g(x)$, where $g(x) \in C[0, \infty)$, which can be written as $C_{\mu}^{m}$ if $F^{(m)} \in C_{\mu}$, where $m \in \mathbb{N}$.

Definition 2 For any function (integrable) $\rho(x) \in C_{\mu}$, the Riemann-Liouville fractional integral operator is defined as

$$
\left\{\begin{array}{l}
J_{t}^{\alpha} \rho(x)=\frac{1}{\Gamma(\alpha)} \int_{0}^{x}(x-t)^{\alpha-1} \rho(t) d t, \text { where } \alpha>0, \quad x>0, \\
J_{t}^{0} \rho(x)=\rho(x) .
\end{array}\right.
$$

Definition 3 The Caputo fractional order derivative is defined as

$$
D_{t}^{\alpha} \rho(x)=J_{t}^{r-\alpha} D_{t}^{r} \rho(x)=\frac{1}{\Gamma(r-\alpha)} \int_{0}^{x}(x-t)^{r-\alpha-1} \rho^{(r)}(t) d t
$$

where, $r-1<\alpha \leq r, r \in \mathbb{N}, x>0$. 
Lemma 1 For the interval $r-1<\alpha \leq r, r \in \mathbb{N}$ and $f \in C_{\mu}^{r} \geq-1$, then

$$
\begin{cases}D_{t}^{\alpha} J_{t}^{\alpha} \rho(x)=\rho(x), & \text { if } x>0, \\ J_{t}^{\alpha} D_{t}^{\alpha} \rho(x)=\rho(x)-\sum_{k=0}^{r-1} \rho^{(k)}\left(0^{+}\right) \frac{x^{k}}{k !}, & \text { if } r-1<\alpha \leq r .\end{cases}
$$

Here we use Caputo fractional derivative because it includes the traditional initial and boundary conditions in the formulation of the physical problems. For more details, see [1-4].

\section{Fractional Reduced Differential Transform Method (FRDTM)}

Taking a two variable function $\breve{w}(x, t)$, where $\breve{w}$ is $k$-times constantly differentiable function with respect to variable $t$ and $x$. The function $\breve{w}(x, t)$ is written as $\breve{w}(x, t)=f(x) . g(t)$, which can be signified as $[20]$

$$
\breve{w}(x, t)=\left(\sum_{i=0}^{\infty} F(i) x^{i}\right)\left(\sum_{j=0}^{\infty} G(j) t^{j}\right)=\left(\sum_{k=0}^{\infty} \breve{W}_{k}(x) t^{k}\right)
$$

Here the function $\breve{w}(x, t)$ is differentiable and analytic, thus the $t$-dimensional form of the function is

$$
\breve{W}_{k}(x)=\frac{1}{\Gamma(\alpha k+1)}\left(\frac{\partial^{\alpha k}}{\partial t^{\alpha k}} \breve{w}(x, t)\right)_{t=t_{0}}
$$

The function $\breve{w}(x, t)$ signifies the novel function and $\breve{W}_{k}(x)$ denotes the transformed function of the proposed method. However, inverse transform of $\breve{W}_{k}(x)$ is specified by

$$
\breve{w}(x, t)=\sum_{k=0}^{\infty} \breve{W}_{k}(x)\left(t-t_{0}\right)^{\alpha k}
$$

Since from equations (2) and (3), one can assume

$$
\breve{w}(x, t)=\sum_{k=0}^{\infty} \frac{1}{\Gamma(\alpha k+1)}\left(\frac{\partial^{\alpha k}}{\partial t^{\alpha k}} \breve{w}(x, t)\right)_{t=t_{0}}\left(t-t_{0}\right)^{\alpha k}
$$

Thus at $t_{0}=0$, equation (4) can be written as

$$
\breve{w}(x, t)=\sum_{k=0}^{\infty} \frac{1}{\Gamma(\alpha k+1)}\left(\frac{\partial^{\alpha k}}{\partial t^{\alpha k}} \breve{w}(x, t)\right)_{t=0} t^{\alpha k} .
$$

Selected basic operations and proofs can be found in [9, 21-24]. Table 1 lists the basic operations of FRDTM. In the table, $\Gamma$ denotes the gamma function where $\Gamma(m+1)=$ $m \Gamma(m), m>0$. 
Table 1: Basic Operations of FRDTM

\begin{tabular}{|l|l|}
\hline \multicolumn{1}{|c|}{ Functional form } & Transformed form \\
\hline$\breve{w}(x, t)$ & $\frac{1}{\Gamma(\alpha k+1)}\left(\frac{\partial^{\alpha k}}{\partial t^{\alpha k}} \breve{w}(x, t)\right)_{t=0}$ \\
\hline$\gamma \breve{w}(x, t) \pm \beta \breve{v}(x, t)$ & $\gamma \breve{W}_{k}(x) \pm \beta \breve{V}_{k}(x)$ where $\gamma$ and $\beta$ are constants \\
\hline$\breve{w}(x, t) \breve{v}(x, t)$ & $\sum_{i=0}^{k} \breve{W}_{j}(x) \breve{V}_{k-i}(x)$ \\
\hline$\breve{w}(x, t) \breve{v}(x, t) \breve{u}(x, t)$ & $\sum_{i=0}^{k} \sum_{j=0}^{i} \breve{W}_{j}(x) \breve{V}_{j}(x) \breve{U}_{k-i}(x)$ \\
\hline$\frac{\partial^{n k}}{\partial t^{n k}} \breve{w}(x, t)$ & $\frac{\Gamma(\alpha k+n \alpha+1)}{\Gamma(\alpha k+1)} \breve{W}_{k+n}(x)$ \\
\hline$\frac{\partial^{n}}{\partial x^{n}} \breve{w}(x, t)$ & $\frac{\partial^{n}}{\partial x^{n}} \breve{W}(x)$ \\
\hline$x^{m} t^{n} \breve{w}(x, t)$ & $x^{m} \breve{W}_{k-n}(x)$ \\
\hline$x^{m} t^{n}$ & $x^{m} \delta(k \alpha-n)$, where $\delta(k \alpha-n)=\left\{\begin{array}{ll}1 & \alpha k=n \\
0 & \alpha k \neq n\end{array}\right\}$ \\
\hline
\end{tabular}

\section{Methodology}

To demonstrate how the FRDTM works, we consider the general form of fractional non-linear non-homogeneous partial differential equation.

$$
L(\breve{w}(x, t))+\Re(\breve{w}(x, t))+N(\breve{w}(x, t))=L(p(x, t))
$$

subject to the initial concentrations

$$
\breve{w}(x, 0)=f(x), \breve{w}_{t}(x, 0)=g(x) .
$$

Here $L=D_{t}^{\alpha}, \Re$ denotes the linear differential operator, $p(x, t)$ is the non-homogeneous source term, whereas $N$ signifies the generalized nonlinear operator.

Using Table 1 and equation (6), we obtain the following

$$
\frac{\Gamma(\alpha k+\alpha+1)}{\Gamma(\alpha k+1)} \breve{W}_{k+1}(x)=P_{k}(x)-\Re\left(\breve{W}_{k}(x)\right)-N\left(\breve{W}_{k}(x)\right)
$$

where $\breve{W}_{k}(x), P_{k}(x), \Re\left(\breve{W}_{k}(x)\right)$ and $N\left(\breve{W}_{k}(x)\right)$ are the transformed forms of the functions $L(\breve{w}(x, t)), L(p(x, t)), \Re(\breve{w}(x, t))$ and $\mathrm{N}(\breve{w}(x, t))$, respectively. From equation (7), we obtain

$$
\breve{W}_{0}(x)=f(x) ; \breve{W}_{1}(x)=g(x)
$$


For successive iterations, we substitute equation (9) into equation (8) to find the iterative values of $\breve{W}_{k}(x)$. Lastly, we employ inverse transformation on all the values of $\left\{\breve{W}_{k}(x)\right\}_{k=0}^{n}$, to get

$$
\breve{w}(x, t)=\sum_{k=0}^{n} \breve{W}_{k}(x) t^{\alpha k}
$$

Moreover, the exact solution is given by $\breve{w}(x, t)=\lim _{n \rightarrow \infty} \breve{w}_{n}(x, t)$.

\section{$5 \quad$ Numerical Applications}

In this section, two examples of KPP equations are considered to validate the efficiency and applicability of FRDTM.

Example 1 Consider the linear time - fractional KPP equation

$$
\frac{\partial^{\alpha} \breve{w}(x, t)}{\partial t^{\alpha}}=\frac{\partial^{2} \breve{w}(x, t)}{\partial x^{2}}-\breve{w}(x, t) \quad x>0, t>0,0<\alpha \leq 1
$$

subject to initial condition

$$
\breve{w}(x, 0)=e^{-x}+x, \quad x \in R
$$

Now, applying FRDTM to equation (11) and (12), we get

$$
\begin{aligned}
\breve{W}_{k+1}(x) & =\frac{\Gamma(\alpha k+1)}{\Gamma(\alpha k+\alpha+1)}\left(\frac{\partial^{2} \breve{W}_{k}}{\partial x^{2}}-\breve{W}_{k}\right), \\
\breve{W}_{0}(x) & =e^{-x}+x .
\end{aligned}
$$

After substituting equation (14) into equation (13), the given recursive values of $\breve{W}_{k}$ are obtained successively:

$$
\begin{aligned}
& \breve{W}_{1}(x)=-\frac{x}{\Gamma(\alpha+1)}, \\
& \breve{W}_{2}(x)=\frac{x}{\Gamma(2 \alpha+1)}, \\
& \breve{W}_{3}(x)=-\frac{x}{\Gamma(3 \alpha+1)} .
\end{aligned}
$$

If we proceed in this manner, the inverse differential transform of $\left\{\breve{W}_{k}(x)\right\}_{k=0}^{\infty}$ is as follows

$$
\breve{w}(x, t)=\sum_{k=0}^{\infty} \breve{W}_{k}(x) t^{\alpha k}=\breve{W}_{0}(x)+\breve{W}_{1}(x) t^{\alpha}+\breve{W}_{2}(x) t^{2 \alpha}+\breve{W}_{3}(x) t^{3 \alpha}+\cdots
$$

Thus, we have the solution of equation (11) in a series form for $\alpha=1$

$$
\breve{w}(x, t)=e^{-x}+x\left(1-t+\frac{t^{2}}{2 !}-\frac{t^{3}}{3 !}+\frac{t^{4}}{4 !}-\cdots\right) .
$$


Hence,

$$
\breve{w}(x, t)=e^{-x}+x e^{-t} .
$$

Table 2 shows the results obtained by FRDTM for two values of $\alpha$. We use different values of $t$ and $x=0.5$. Also, exact solution for $\alpha=1$ is given which shows thatas the number of iterations increases, the accuracy improves and approaches to the exact solution. However, as $t$ approaches to 0 the error is reducing and if $t>1$, the error will be increasing.

Table 2: Results of FRDTM for Different Values of $\alpha$

\begin{tabular}{|c|c|c|c|c|c|}
\hline $\boldsymbol{x}$ & $\boldsymbol{t}$ & $\alpha=0.5$ & $\alpha=1.0$ & $\alpha=1.0$ & Error \\
\hline \multirow{6}{*}{$\mathbf{0 . 5}$} & & FRDTM & FRDTM & Exact & \\
\cline { 2 - 6 } & 0.1 & 0.9687240876 & 1.058949410 & 1.058949369 & $4.1 \mathrm{E}-08$ \\
\cline { 2 - 6 } & 0.2 & 0.9305756408 & 1.015897326 & 1.015896036 & $1.29 \mathrm{E}-06$ \\
\cline { 2 - 6 } & 0.3 & 0.9082074260 & 0.9769494100 & 0.9769397701 & $9.6399 \mathrm{E}-06$ \\
\cline { 2 - 6 } & 0.4 & 0.8945525506 & 0.9417306600 & 0.9416906827 & $3.99773 \mathrm{E}-05$ \\
\cline { 2 - 6 } & 0.5 & 0.8871076194 & 0.9099160766 & 0.9097959895 & 0.000120087 \\
\cline { 2 - 6 } & 0.6 & 0.8847035389 & 0.8812306600 & 0.8809364777 & 0.000294182 \\
\cline { 2 - 6 } & 0.7 & 0.8867128478 & 0.8554494100 & 0.8548233116 & 0.000626098 \\
\cline { 2 - 6 } & 0.8 & 0.8927700203 & 0.8323973266 & 0.8311951417 & 0.001202185 \\
\cline { 2 - 6 } & 0.9 & 0.9026510841 & 0.8119494100 & 0.8098154895 & 0.00213392 \\
\cline { 2 - 6 } & 1.0 & 0.9162146869 & 0.7940306600 & 0.7904703803 & 0.00356028 \\
\hline
\end{tabular}

Example 2 Consider the non-linear time-space fractional KPP equation

$$
\frac{\partial^{\alpha} \breve{w}(x, t)}{\partial t^{\alpha}}-\frac{\partial^{2 \beta} \breve{w}(x, t)}{\partial x^{2 \beta}}+2 \breve{w}^{3}=0, \quad t>0, \alpha>0, \beta \leq 1
$$

with the initial condition

$$
\breve{w}(x, 0)=x^{2} .
$$

The following recurrence relation is obtained by applying FRDTM to equation (21)

$$
\breve{W}_{k+1}(x)=\frac{\Gamma(\alpha k+1)}{\Gamma(\alpha k+\alpha+1)}\left(\frac{\partial^{2 \beta} \breve{W}_{k}}{\partial x^{2 \beta}}-2 \sum_{i=0}^{k} \sum_{j=0}^{i} \breve{W}_{i-j} \breve{W}_{j} \breve{W}_{k-i}\right) .
$$

Now, applying FRDTM to the initial condition (22), we get

$$
\breve{W}_{0}(x)=x^{2} .
$$

Upon substituting the above equation (24) in equation (23), the given recursive values of $\breve{W}_{k}$ are obtained successively:

$$
\breve{W}_{1}(x)=\frac{2\left(\left(\frac{1}{\Gamma(3-2 \beta)}\right) x^{2-2 \beta}\right)-2 x^{6}}{\Gamma(\alpha+1)},
$$




$$
\begin{aligned}
\breve{W}_{2}(x) & =\frac{1}{\Gamma(2 \alpha+1)} \\
& {\left[\Gamma(\alpha+1)\left(\frac{2\left(\frac{1}{\Gamma(3-2 \beta)}\left(\frac{\Gamma(3-2 \beta)}{\Gamma(3-4 \beta)}\right)\right) x^{2-4 \beta}-1440\left(\left(\frac{1}{\Gamma(7-2 \beta}\right) x^{6-2 \beta}\right)}{\Gamma(\alpha+1)}\right)\right] } \\
& -\frac{5}{\Gamma(2 \alpha+1)}\left[\Gamma(\alpha+1)\left(\frac{2\left(\left(\frac{1}{\Gamma(3-2 \beta)}\right) x^{2-2 \beta}\right)-2 x^{6}}{\Gamma(\alpha+1)}\right)\left(x^{4}\right)\right] \ldots
\end{aligned}
$$

and so on.

If we proceed in this manner, the inverse differential transform of $\left\{\breve{W}_{k}(x)\right\}_{k=0}^{\infty}$ is as follows

$$
\breve{w}(x, t)=\sum_{k=0}^{\infty} \breve{W}_{k}(x) t^{\alpha k}=\breve{W}_{0}(x)+\breve{W}_{1}(x) t^{\alpha}+\breve{W}_{2}(x) t^{2 \alpha}+\breve{W}_{3}(x) t^{3 \alpha}+\cdots
$$

The same solution are also obtained by Gupreel [5] using Homotopy Pertubation Method (HPM).

Table 3 shows the result obtained by FRDTM and compared with HPM for different values of $\alpha$. We use different values of $t$ and $x=\beta=0.5$. The results show that FRDTM requires less computation. Since it gives an approximate series solution in just few iterations without making polynomials as in HPM [5].

Table 3: Comparison of FRDTM with HPM for $\alpha=0.5$ and 1.0

\begin{tabular}{|c|c|c|c|c|c|}
\hline $\boldsymbol{x}$ & $\boldsymbol{t}$ & $\alpha=0.5$ & $\alpha=0.5$ & $\alpha=1.0$ & $\alpha=1.0$ \\
\hline \multirow{7}{*}{$\mathbf{0 . 5}$} & & $H P M$ & $F R D T M$ & $H P M$ & $R D T M$ \\
\cline { 2 - 6 } & 0.1 & 0.5956740474 & 0.7218459223 & 0.3468750 & 0.3531835938 \\
\cline { 2 - 6 } & 0.2 & 0.7388569261 & 0.9912006759 & 0.4437500 & 0.4689843750 \\
\cline { 2 - 6 } & 0.3 & 0.8487250131 & 1.227240638 & 0.5406250 & 0.5974023438 \\
\cline { 2 - 6 } & 0.4 & 0.9413480949 & 1.446035594 & 0.6375000 & 0.7384375000 \\
\cline { 2 - 6 } & 0.5 & 1.022950668 & 1.653810042 & 0.7343750 & 0.8920898438 \\
\cline { 2 - 6 } & 0.6 & 1.096725034 & 1.853756283 & 0.8312500 & 1.058359375 \\
\cline { 2 - 6 } & 0.7 & 1.164567564 & 2.047770688 & 0.9281250 & 1.237246094 \\
\cline { 2 - 6 } & 0.8 & 1.227713852 & 2.237088851 & 1.0250000 & 1.428750000 \\
\cline { 2 - 6 } & 0.9 & 1.287022142 & 2.422569016 & 1.1218750 & 1.632871094 \\
\cline { 2 - 6 } & 1.0 & 1.343117318 & 2.604836067 & 1.2187500 & 1.849609375 \\
\hline
\end{tabular}

\section{Conclusion}

Numerical examination of fractional KPP equations have been successfully studied in this paper. The proposed technique is effective in obtaining the solutions of linear and non-linear 
time-space fractional differential equations. The results obtained can be compared with the classical solution of the problem for $\alpha=1$ and with HPM [25]. This shows that Fractional Reduced differential transform method (FRDTM) is efficient with low computational cost and converge faster when compared with HPM. Since FRDTM provides series form solution in less iterations instead of making polynomials as in HPM [25].

\section{Acknowledgement}

The authors are grateful to the reviewers and the editor for their helpful comments and suggestions which indeed improved the quality of this paper.

\section{References}

[1] Podlubny, I. Fractional Differential Equations. San Diego: Academic Press. 1999.

[2] Miller, K. S. and Ross, B. An Introduction to the Fractional Calculus and Fractional Differential Equations. New York: Wiley. 1993.

[3] Hilfer, R. Applications of Fractional Calculus in Physics. Singapore: World Scientific. 2000.

[4] Klafter, J., Lim, S. C. and Metzler, R. Fractional Dynamics: Recent Advances. Singapore: World Scientific. 2012.

[5] Iyiola, O. S., Ojo, G. O. and Mmaduabuchi, O. The fractional Rosenau-Hyman model and its approximate solution. Alexandria Engineering Journal. 2016. 55(2): 1655-1659.

[6] Zaidan, L. I., and Darvishi, M. T. Numerical simulation for fractional nonlinear $(1+1)$ dimensional Biswas-Milovic equation. Optik-International Journal for Light and Electron Optics136. 2017. 390-402.

[7] Javed, I., Ahmad, A., Hussain, M. and Iqbal, S. Some Solutions of Fractional Order Partial Differential Equations Using Adomian Decomposition Method. arXiv preprint arXiv: 1712.09207. 2017.

[8] Singh, B. K. and Kumar, P. Fractional variational iteration method for solving fractional partial differential equations with proportional delay. International Journal of Differential Equations. 2017. 1-11.

[9] Kerstin, K, Y. Selcuk University: Ph.D. Thesis. 2010.

[10] Keskin Y., and Oturanc G. Reduced differential transform method for partial differential equations. Int J Nonlinear Sci. Numer. Simul. 2009. 10(6): 741-749.

[11] Keskin Y. and Oturanc G. Reduced differential transform method: a new approach to fractional partial differential equations. Nonlinear Sci. Lett. 2010. 1: 61-72.

[12] Abuasad, S., Moaddy, K. and Hashim, I. Analytical treatment of two-dimensional fractional Helmholtz equations. Journal of King Saud University-Science. 2018.

[13] Singh, B. K. and Kumar, P. Extended Fractional Reduced Differential Transform for Solving Fractional Partial Differential Equations with Proportional Delay. International Journal of Applied and Computational Mathematics. 2017. 3(1): 631-649. 
[14] Chaurasia, R. K., Mathur, V., Pareekh, R. L., Tamsir, M. and Srivastava, V. K. A computational modelling of micro strip patch antenna and its solution by RDTM. Alexandria Engineering Journal. 2017. 57(3): 1877-1881.

[15] Singh, B. K. and Kumar, P. FRDTM for numerical simulation of multi-dimensional, timefractional model of Navier-Stokes equation. Ain Shams Engineering Journal. 2018. 9(4): $827-834$

[16] Babaei, A. On Analytical Approximate Solution of the Fractional Type Rosenau-Hyman Equation. Fundamenta Informaticae. 2017. 151(1-4): 135-143.

[17] Singh, B. K., and Kumar, P. A New Approximate Solution of Time-Fractional, Nonlinear Schrodinger Equations Using Fractional Reduced Differential Transformation. arXiv preprint arXiv: 1611.071712016.

[18] Tamsir, M. Srivastava, V. K. Analytical study of time-fractional order Klein-Gordon equation. Alexandria Engineering Journal. 2016. 55: 561-567

[19] Arshad, M., Lu, D., and Wang, J. (N+1)-dimensional fractional reduced differential transform method for fractional order partial differential equations. Communications in Nonlinear Science and Numerical Simulation. 2017. 48: 509-519.

[20] Rawashdeh, M. S. A reliable method for the space-time fractional Burgers and timefractional Cahn-Allen equations via the FRDTM. Advances in Difference Equations. 2017. Article number 99.

[21] Rawashdeh, M. Improved approximate solutions for nonlinear evolutions equations in mathematical physics using the reduced differential transform method. Journal of Applied Mathematics and Bioinformatics. 2013. 3(2): 1-14.

[22] Rawashdeh, M. Using the reduced differential transform method to solve nonlinear PDEs arises in biology and physics. World Appl. Sci. J. 2013. 23(6): 1037-1043.

[23] Rawashdeh, M. and Obeidat. On finding exact and approximate solutions to some PDEs using the reduced differential transform method. Appl. Math. Inf. Sci. 2014. 8(4): 21712176.

[24] Rawashdeh, M. Approximate solutions for coupled systems of nonlinear PDES using the reduced differential transform method. Math. Comput. Appl. 2014. 19(2): 161-171.

[25] Gupreel, K. A. The homotopy perturbation method applied to the nonlinear fractional Kolmogrov-Petrovskii-Piskunov equations. Appl. Math. 2011. 24: 1428-1434. 